\title{
Caracterización molecular de aislamientos invasores de Streptococcus pneumoniae resistentes a la penicilina recuperados de pacientes adultos
}

\author{
Jaime Moreno, Elizabeth Castañeda \\ Grupo de Microbiología, Instituto Nacional de Salud, Bogotá, D.C., Colombia.
}

La resistencia de Streptococcus pneumoniae a la penicilina está asociada con la dispersión de ciertos clones internacionales. En Colombia, se ha establecido en la población infantil la presencia y circulación de los clones 1 -España ${ }^{23 F}, 2-E_{s p a n ̃ a}{ }^{6 B}, 3-E_{s p a n ̃ a}{ }^{9 V}$ y $25-$ Colombia $^{23 F}$. El objetivo de este trabajo fue evaluar las relaciones clonales entre 80 aislamientos invasores de $S$. pneumoniae con susceptibilidad disminuida a la penicilina recuperados de pacientes adultos. El análisis genotípico incluyó la electroforesis en campo pulsado (PFGE) del ADN cromosómico digerido con la enzima Sma I, la restricción de los genes de las proteínas de unión a la penicilina (PBP) 1a, 2b y 2x y la distribución de las familias de la proteína de superficie A (PspA) por dotblot y PCR. Los resultados mostraron que $2,5 \%$ de los aislamientos estaban genéticamente relacionados con el clon 1-España ${ }^{23 F}, 10 \%$ con el clon 2 -España ${ }^{6 B}$ y $37,5 \%$ con el clon 3España $^{9 v}$. Además, $14 \%$ de los aislamientos de tipo capsular $23 \mathrm{~F}$ y con resistencia intermedia a la penicilina se relacionaron con el clon 25-Colombia ${ }^{23 F}$. Estos datos evidencian la circulación de clones internacionales, así como de un clon colombiano en la población adulta y señalan su importancia en la prevalencia de la resistencia a la penicilina en nuestro medio.

Palabras clave: Streptococcus pneumoniae, susceptibilidad disminuida a la penicilina, aislamientos invasores, proteínas de unión a penicilina, proteína de superficie A, clones.

\section{Molecular characterization of invasive isolates of Streptococcus pneumoniae with diminished susceptibility to penicillin}

Streptococcus pneumoniae penicillin resistance is associated with the international dispersion of specifically identified strains. In Colombia, the presence and circulation of resistant strains Spain $^{23 F_{-}}$1, Spain ${ }^{6 B_{-}}-2$, Spain ${ }^{9 \mathrm{~V}}$ and Colombian ${ }^{23 \mathrm{~F}}-25$ has been demostrated in children less than 5 years old. Strain identities were established for 80 penicillin resistant $S$. pneumoniae isolates recovered from Colombian adult patients. Three approaches to genotypic characterization of the strains wrere used: a) chromosomal DNA was digested with Sma I, and the products subjected to pulse-field gel electrophoresis (PFGE); b) restriction enzyme fagment comparison of the penicillin binding protein genes ( $p b p 1 \mathrm{a}, 2 \mathrm{~b}$ and $2 \mathrm{x}$ ), and $\mathrm{c}$ ) pneumococcal surface protein $\mathrm{A}(\mathrm{PspA})$ family. The results showed that $2.5 \%$ of the isolates were genetically related to Spain ${ }^{23 \mathrm{~F}}-1$ clone, $10 \%$ to Spain ${ }^{6 \mathrm{~B}}-2$ clone, $37.5 \%$ to Spain ${ }^{9 \mathrm{~V}}-3$, in addition, $14 \%$ of the capsular type $23 \mathrm{~F}$ isolates were related to the Colombia ${ }^{23 F}-25$ clone and demonstrated an intermediate level resistance to penicillin. Wide spread circulation of international strains as well as a Colombian strain highlighted the importance of these clones in maintaining the prevalence of resistance to penicillin in Colombia.

Key words: Streptococcus pneumoniae, disminished susceptibility to penicillin, invasive isolates, penicillin binding proteins, pneumococcal surface protein $A$, clones.

Correspondencia:

Elizabeth Castañeda, Grupo de Microbiología, Instituto Nacional de salud, Av. Calle 26 No. 51-60, Bogotá, D.C., Colombia

ecastaneda@ins.gov.co

Recibido: 06/11/02; aceptado: 31/02/03
Las infecciones ocasionadas por Streptococcus pneumoniae causan gran morbilidad y mortalidad en niños, en adultos en edad avanzada y en individuos con condiciones debilitantes (1). $S$. pneumoniae es el agente más prevalente en otitis 
media, es responsable de la mayoría de los casos de neumonía adquirida en la comunidad y uno de los tres patógenos que más comúnmente causan meningitis bacteriana (1). Sin embargo, la frecuencia de estas infecciones en países en vía de desarrollo no se conoce debido a problemas de diagnóstico y al inadecuado informe de casos (2). El problema se incrementa al reconocer que $S$. pneumoniae ha adquirido resistencia a la mayoría de los agentes antimicrobianos (3). Los aislamientos resistentes a la penicilina se han descrito en todo el mundo, con una incidencia que supera el $50 \%$ en países como Japón, Tailandia, Vietnam y Corea (4), 60\% en España (5), 35\% en Estados Unidos (6) y $11 \%$ en Canadá (7); en América Latina, la incidencia varía del $15 \%$ en Brasil hasta el $48 \%$ en México $(8,9)$. En Colombia, la resistencia a penicilina en el año 2000 para aislamientos invasores de niños menores de 5 años fue del $36 \%$ (10).

La resistencia a la penicilina involucra la producción de formas alteradas de las proteínas de unión a la penicilina (PBP) 1a, 2b y 2x, las cuales son enzimas involucradas en el ensamblaje y metabolismo del peptidoglicano de la pared celular bacteriana $(11,12)$. El incremento de la resistencia a la penicilina es el resultado de la adquisición de genes de resistencia dentro de líneas clonales susceptibles, la transmisión de genes de resistencia a nuevos grupos clonales y la dispersión de clones resistentes, un fenómeno global y ampliamente documentado $(13,14)$. Actualmente, la red de epidemiología molecular del neumococo (The Pneumococcal Molecular Epidemiology Network, PMEN) reconoce más de 20 clones diferentes (14). La caracterización molecular de $S$. pneumoniae resistente a la penicilina requiere el empleo de técnicas que evalúen las relaciones genéticas de los aislamientos, el análisis de los genes $p b p$ y se recomienda la aplicación de, por lo menos, otra técnica de tipificación usando genes específicos de especie o altamente conservados para establecer y confirmar relaciones clonales $(15,16)$. Los genes de la proteína de superficie A ( $p s p \mathrm{~A})$, presentes en todos los aislamientos de $S$. pneumoniae constituyen un buen marcador molecular (17). Estas proteínas se clasifican en tres familias y se pueden tipificar con el uso de antisueros policlonales anti-PspA o por la amplificación de los genes que la codifican con la reacción en cadena de la polimerasa $(18,19)$.

En Colombia, en 1994, a través del proyecto del Sistema Regional de Vacunas (SIREVA) de la Organización Panamericana de la Salud se inició un proyecto regional para vigilancia de la distribución de los tipos capsulares y de la susceptibilidad antimicrobiana de los aislamientos de $S$. pneumoniae, causantes de enfermedad invasora en niños menores de 5 años (20). Los estudios moleculares de los aislamientos invasores con susceptibilidad disminuida a la penicilina (SDP) demostraron la circulación de los clones internacionales 1-España ${ }^{23 F}$, 2-España ${ }^{6 B}$ y 3-España ${ }^{9 v}$ y de un grupo clonal con serotipo 23F denominado como clon 'colombiano' $(20,21)$ y actualmente reconocido por la PMEN como el clon 25-Colombia ${ }^{23 F}$ (minutas de la sexta reunión del PMEN, ICAAC 2002), asociados con ciertos perfiles de las PBP (21). De igual forma, se determinó que el $95 \%$ de 40 aislamientos pertenecían a las familias $1(62,5 \%)$ y $2(35 \%)$ de la PspA, lo cual demuestra que esta proteína es muy homogénea y puede ser un buen inmunógeno en nuestro medio (22). Con los programas de meningitis bacteriana aguda (MBA) e infección respiratoria aguda (IRA) del Grupo de Microbiología del Instituto Nacional de Salud, se inició la vigilancia de $S$. pneumoniae aislado de procesos invasores en población mayor de 5 años y se pudo establecer que el $24,7 \%$ de los aislamientos recuperados de pacientes mayores de 16 años presentaban SDP asociada con los tipos capsulares $14,23 \mathrm{~F}, 9 \mathrm{~V}, 6 \mathrm{~B}$ y $19 \mathrm{~F}$ (23). El objetivo de este trabajo fue el de realizar una vigilancia molecular de los aislamientos invasores de $S$. pneumoniae con SDP recuperados de la población mayor de 14 años para determinar los tipos clonales prevalentes y circulantes en esta población.

\section{Materiales y métodos}

\section{Aislamientos y características de los pacientes}

Se estudiaron 80 aislamientos invasores de $S$. pneumoniae con SDP recuperados de pacientes adultos (mayores de 14 años). Los aislamientos 
fueron recibidos en el Grupo de Microbiología, como parte de los programas de vigilancia por el laboratorio de MBA e IRA durante el periodo comprendido entre junio de 1994 y diciembre del 2001. Los aislamientos estaban serotipificados con la reacción capsular de quellung y se conocían sus patrones de resistencia expresada como la concentración inhibitoria mínima (CIM) en $\mathrm{mg} / \mathrm{ml}$ a la penicilina, ceftriaxona, eritromicina, cloranfenicol, trimetroprim-sulfametoxazol, vancomicina y tetraciclina, determinada de acuerdo con las recomendaciones del Comité Nacional de Estándares para el Laboratorio Clínico (National Committee for Clinical Laboratory Standards, NCCLS) (24).

Los pacientes tenían de 15 a 89 años; el 50\% eran hombres y procedían de diferentes regiones del país (Antioquia, Bogotá, Risaralda, Santander, Valle y Norte de Santander). Los aislamientos se obtuvieron de hemocultivo (58,8\%), líquido cefalorraquídeo $(32,5 \%)$ y de otros líquidos corporales (8,7\%). El 58,8\% de los pacientes tenían diagnóstico de neumonía, $32,5 \%$ de meningitis y $8,7 \%$ de otros procesos invasores (sepsis, artritis infecciosa). Los principales tipos capsulares de los aislamientos estudiados fueron el 14 (37\%), seguido por el $23 \mathrm{~F}(19 \%), 6 \mathrm{~B}(12 \%)$ y $9 \mathrm{~V}(9 \%)$; el restante $23 \%$ se distribuyó entre 11 serotipos y 3 aislamientos no serotipificables. En 38 (47,5\%) aislamientos se determinó resistencia intermedia a la penicilina $(\mathrm{CIM}=0,125-1,0 \mu \mathrm{g} / \mathrm{ml})$ y en 42 $(52,5 \%)$ resistencia alta $(\mathrm{CIM} \geq 2,0 \mu \mathrm{g} / \mathrm{ml})$.

\section{Cepas de control y referencia}

Para la PFGE se emplearon la cepa acapsular y sensible a antibióticos R6 (donada por Alexander Tomasz, Rockefeller University, Nueva York), utilizada como marcador interno de peso molecular y de las condiciones de corrido electroforético y los clones 1-España ${ }^{23 F}$, 2-España ${ }^{6 \mathrm{~B}}$ y 3-España ${ }^{9 \mathrm{~V}}$ (sumunistrados por el Centro Nacional de Streptococcus, NCS, Alberta, Canadá), como referencia para la asignación de los patrones. También se emplearon los aislamientos colombianos INS Spn 13, serotipo 23F, Spn E296 y Spn E-301 serotipo 14, Spn E-226 serotipo 6B, Spn E-147 serotipo 23F y Spn E-115 serotipo 19F. Para la determinación de las familias de PspA por las técnicas de PCR y dot blot las cepas de referencia fueron: B69739 y EF10197 (familia 1) y AC122, ATCC6303, BG11707 (familia 2) (donadas por David Briles, Alabama University).

\section{PFGE}

Se realizó según el protocolo de Soares y col. (25) y las modificaciones de Vela y col. (21). Brevemente, los aislamientos se cultivaron en caldo Todd-Hewitt suplementado hasta alcanzar la fase logarítmica de crecimiento. Las células fueron embebidas en discos de agarosa de bajo punto de fusión (Bio-Rad) y fueron digeridas con la enzima de restricción Smal (Promega) a una concentración de $20 \mathrm{U} / \mathrm{ml}$; los fragmentos de restricción se separaron en geles de agarosa al $1 \%$ (Bio-Rad) por electroforesis en campo pulsado usando el equipo CHEFF II (Bio-Rad). Los geles se colorearon con bromuro de etidio y se visualizaron con luz ultravioleta. Los patrones de las bandas se asignaron visualmente y se clasificaron según los criterios de Tenover (26); igualmente, se analizaron con el programa de cómputo Diversidad (Bio-Rad).

\section{Determinación del polimorfismo de los genes pbp 1a, $2 b$ y $2 x$}

La extracción del ADN se realizó por el método de Pitcher y col. (27). Para la amplificación de los genes $p b p 1 \mathrm{a}, 2 \mathrm{~b}$ y $2 \mathrm{x}$ por PCR se utilizaron los iniciadores y protocolo previamente descritos (23). Los fragmentos amplificados se digirieron con 5 U de la enzima Hinf I (Promega), se separaron en geles de poliacrilamida al $8 \%$, se colorearon con bromuro de etidio y se visualizaron con luz ultravioleta. Los perfiles se asignaron según el número y posición de las bandas, teniendo en cuenta los informados por Vela y col. (21).

\section{Determinación de las familias PspA por PCR y dot-blot}

Para la determinación de las familias de PspA por PCR se usaron los iniciadores informados por Briles y col. (28) y la técnica utilizada por Vela y col. (22). Los productos se corrieron en geles de agarosa al $0,8 \%$ y se visualizaron con luz ultravioleta. Las familias se asignaron por la presencia de la banda especifica para cada familia (22). Para la técnica de dot-blot se realizó la 
extracción de proteínas totales, se determinó su concentración por el método de ácido bisincónico y sulfato de cobre pentahidratado II (29) y se usó la metodología descrita por Briles y col. (28) y Vela y col. (22). La asignación de las familias se hizo por la presencia de una reacción de color en las membranas, comparándolas con las obtenidas con los controles (22).

\section{Resultados}

\section{Patrones electroforéticos}

Con la técnica de electroforesis en campo pulsado se obtuvieron de 10 a 16 fragmentos, con pesos moleculares de 26,3 a 455,9 kb. Según la interpretación sugerida por Tenover, los 80 aislamientos se agruparon en 29 patrones definidos por las letras de la $A$ hasta la $Z$ y las dobles letras $A B-A E$ con los subtipos $A 1, A 2, B 1$ -B11, C1-C10 y D1-D4. El análisis de los resultados se realizó de acuerdo con su distribución por serotipo.

\section{Serotipo 23F}

Los 15 aislamientos serotipo $23 \mathrm{~F}$ se distribuyeron en 4 patrones electroforéticos: el $A$, conformado por los subtipos A1 y A2, cada uno con un aislamiento y relacionados con el clon internacional 1-España ${ }^{23 F}$ (relación genética mayor de $75 \%$ ); ambos presentaron alta resistencia a la penicilina
( $\mathrm{CIM} \geq 2,0 \mathrm{mg} / \mathrm{ml}$ ), cloranfenicol, trimetroprim-sulfa y tetraciclina. El patrón $C$ se subdividió en 10 subtipos (C1-C10) y estaba conformado por 11 aislamientos $(13,8 \%)$, con un porcentaje de similitud mayor de $80 \%$ con la cepa INS Spn E147 empleada como referencia del clon 25-Colombia ${ }^{23 F}$, todos con un nivel de resistencia intermedio a la penicilina y variable a trimetroprim-sulfa y tetraciclina (figura 1). Los dos aislamientos restantes presentaron patrones electroforéticos diferentes denominados como $\mathrm{L}$ y M.

\section{Serotipo 14 y $9 \mathrm{~V}$}

Los 30 aislamientos serotipo 14 y los 7 serotipo $9 \mathrm{~V}$ se estudiaron en conjunto debido a que entre ellos es un evento frecuente el intercambio de genes capsulares por transformación genética. Por PFGE se obtuvieron 8 patrones electroforéticos designados con las letras $\mathrm{B}$ a K; 30 aislamientos conformaron el patrón $B$ y 11 subtipos de $B$ (B1$B 11)$ con un corrido electroforético asociado con el del clon 3-España ${ }^{9 v}$. De estos 30 aislamientos, 8 tenían patrón $\mathrm{B}$ y fueron idénticos al clon 3,12 aislamientos con patrón B3 presentaron un porcentaje de similitud mayor de $95 \%$ con el clon y para 10 aislamientos el porcentaje fue mayor de $75 \%$ (figura 2). De los aislamientos con patrón B, $27(90 \%)$ presentaron alta resistencia a la penicilina y $18(60 \%)$ tenían un perfil de
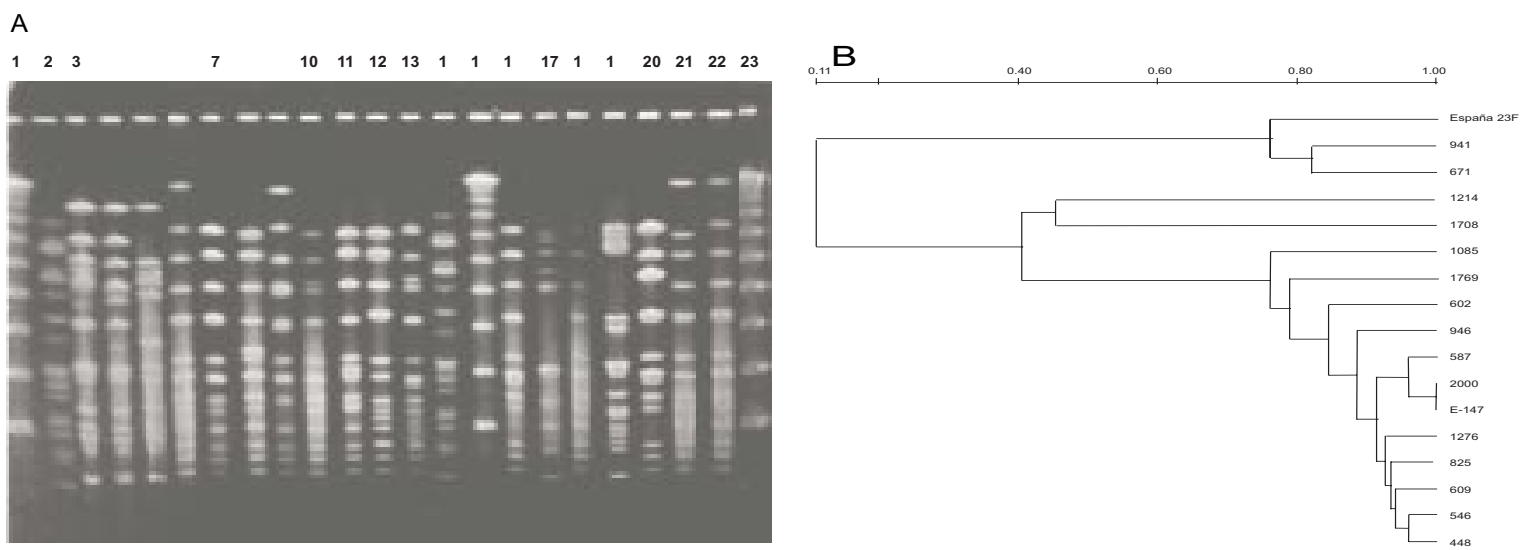

Figura 1. A: patrones de PFGE de los aislamientos de $S$. pneumoniae serotipo $23 \mathrm{~F}$ con el empleo de la enzima de restricción Sma I. Carriles 1, 15 y 23, marcador de peso molecular $1 \mathrm{~kb}$. Carriles 2 y 14, cepa control R6. Carril 3, clon 1- España ${ }^{23 F}$ (patrón A). Carriles 4 y 5 , los aislamientos 941 y 671 con patrón A1 y A2. Carriles 6 y 22, clon 25-Colombia ${ }^{23 F}$ (patrón C), carriles 7 a 13,16, 18, 20, 21, aislamientos 448 patrón C1, 546 patrón C3, 587 patrón C2, 602 patrón $C 7,609$ patrón C5, 825 patrón C4, 946 patrón C6, 1085 patrón C9, 1276 C10, 1769 patrón C8 y 2000 patrón C, respectivamente. Carriles 17 y 19,1214 patrón M y 1708 patrón L, respectivamente. B: dendrograma de los aislamientos de S. pneumoniae serotipo $23 \mathrm{~F}$. 
A

1234567891011121314151617181920212223242526

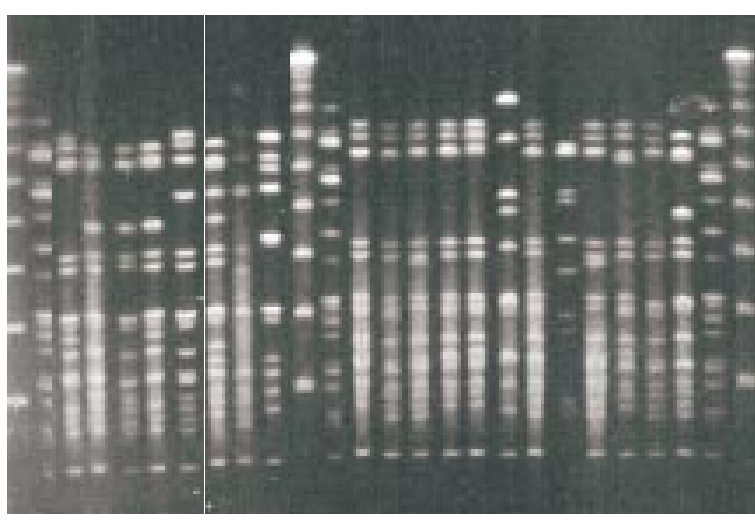

C

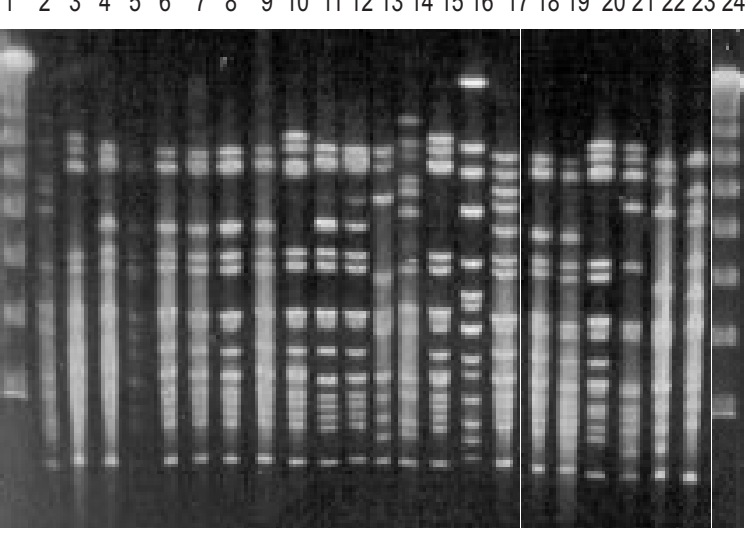

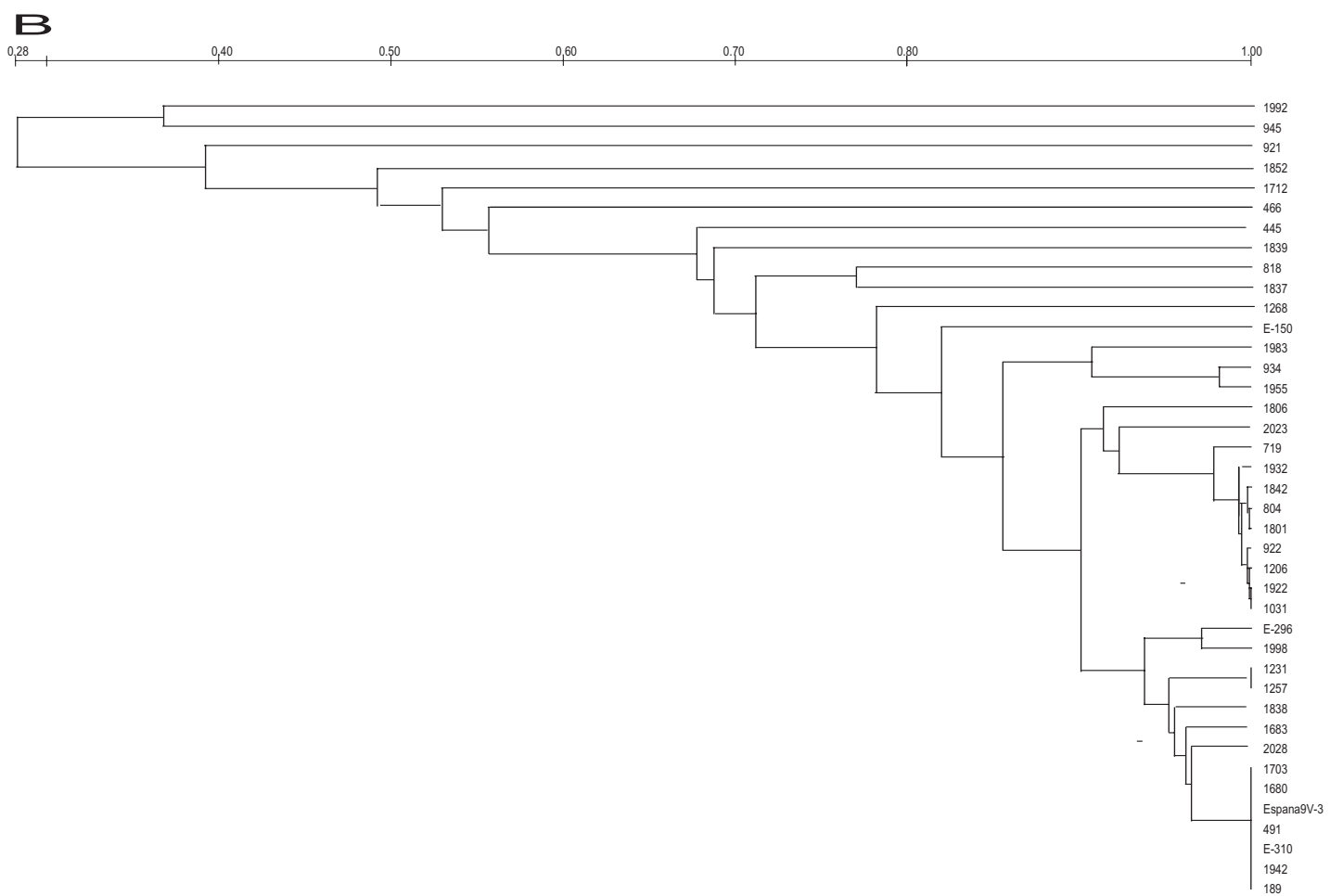

Figura 2. Patrones de PFGE (A y B) de los aislamientos de $S$. pneumoniae serotipo 14 y $9 \mathrm{~V}$ con el empleo de la enzima de restricción Sma I. En A: carriles 1, 11 y 26, marcador $1 \mathrm{~Kb}$. Carriles 2, 12 y 25, cepa control R6. Carril 13 clon 3-España ${ }^{9 v}$ (patrón B). Carriles 14 y 23, aislamientos INS 189 e E-310 (patrón B). Carril 22, INS E-150 (patrón B6). Carriles 3, 15 a 17 y 19, aislamientos 1838, 491, 1231, 1257 y 1942 con patrón B. Carriles 4 a 6 y 24, aislamientos 1922, 1955, 2023 y 934 con patrón B3. Carriles 7, 8, 10 y 21, 1998 patrón B1, 1983 patrón B7, 466 patrón B9 y 2028 patrón B2. Carriles 9, 18 y 20, 445, 1852 y 1992 con patrones I, J y K, respectivamente. En B: Carriles 1 y 24, marcador 1 kb. Carriles 2 y 14, cepa control R6. Carril 3, clon 3-España ${ }^{9 v}$. Carriles 10,15 y 20, aislamientos 1703,1680 y 1683 con patrón B. Carriles 4 a $8,11,18$ y 19 , aislamientos 719, 804, 922, 1031, 1206, 1801, 1842 y 1932 con patrón B3. Carriles 9, 12, 21 y 22, 1268 patrón B6, 1806 patrón B4, 1837 patrón B5 y 818 patrón B8. Carriles 13, 16, 17 y 23, aislamientos 921, 1712, 1839 y 945 con patrones $E$, F, G y H, respectivamente. No se muestran los patrones electroforéticos de los aislamientos 1851 (patrón B10) y 2001 (patrón B11). En C: dendrograma de los aislamientos de $S$. pneumoniae serotipo 14 y $9 \mathrm{~V}$. 
susceptibilidad antimicrobiana idéntico al del clon 3-España ${ }^{9 v}$, es decir, resistentes a penicilina y trimetroprim-sulfa, susceptibilidad intermedia a ceftriaxona y sensibles a eritromicina, cloranfenicol, vancomicina y tetraciclina.

\section{Serotipo 6B}

Los 10 aislamientos serotipo 6B presentaron un sólo patrón D y 4 subtipos (D1-D4), los cuales están relacionados con los perfiles electroforéticos del clon 2- España ${ }^{6 B}$; el porcentaje de similitud para el patrón $\mathrm{D}$ con respecto al clon 2 fue de $100 \%$ para D1, de $95 \%$ para D3, de $92 \%$ y mayor de $75 \%$ para los patrones D2 y D4 (figura 3). Todos los aislamientos fueron resistentes a penicilina, trimetroprim-sulfa y cloranfenicol, 90\% a tetraciclina, $80 \%$ a ceftriaxona y $70 \%$ a eritromicina.

\section{Aislamientos con otros serotipos}

Dieciocho aislamientos se distribuyeron en 11 serotipos y 17 patrones electroforéticos. Con excepción de 2 aislamientos no serotipificables, que presentaron el patrón único $\mathrm{Q}$, los restantes presentaron diferentes patrones electroforéticos. Al comparar estos patrones no se encontró ninguna asociación con respecto a los clones internacionales referenciados. Sin embargo, la mayoría de estos aislamientos (95\%) presentaban una resistencia intermedia a la penicilina.

\section{Perfiles de restricción de los genes pbp 1a, $2 b$ y $2 x$}

Por medio de la restricción de los genes $p b p$ con la enzima Hinf I se determinaron 9 patrones de restricción para pbp 2b, 15 para pbp 2x y 3 para pbp 1a, que conformaron 25 perfiles, de los cuales los más frecuentemente encontrados fueron el I $(21,25 \%)$, el II $(16,25 \%)$ y el III $(21,25 \%)$; los 22 perfiles restantes (IV - XXV) se distribuyeron entre 32 aislamientos. En forma general se observó que existía una asociación entre serotipos, patrones de PFGE y perfiles de las PBP, el perfil I se observó en los aislamientos de tipo capsular 23F con patrón $A$, en los serotipos 14 y $9 \mathrm{~V}$ con patrón $B$ y en algunos subtipos diferentes del B3. El perfil II se encontró casi exclusivamente asociado con los aislamientos $23 \mathrm{~F}$ con patrón $\mathrm{C}$. De igual manera, el perfil III sólo se encontró en los aislamientos con serotipo 14 y particularmente en el subtipo $B$ 3. Los perfiles VI y VII se observaron en los aislamientos $6 \mathrm{~B}$, patrón $\mathrm{D}$. Sólo 2 perfiles se encontraron en dos o más aislamientos con serotipos diferentes, el $X V I$, patrones $\mathrm{K}, \mathrm{O}, \mathrm{AB}$, $A C$ y $A E$, y el perfil VIII en 2 aislamientos no serotipificables.
A

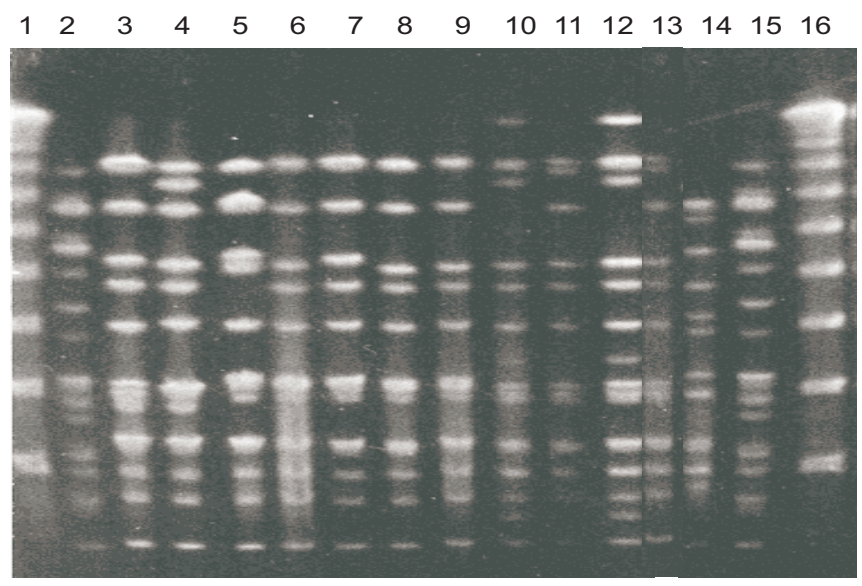

B

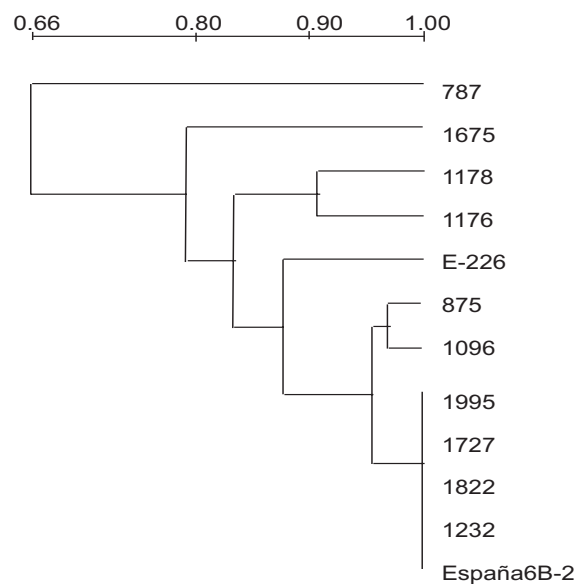

Figura 3. A: Patrones de PFGE de los aislamientos de $S$. pneumoniae serotipo $6 \mathrm{~B}$ con el empleo de la enzima de restricción Sma I. Carriles 1 y 16, marcador de peso molecular 1 kb. Carriles 2 y 15, cepa control R6. Carril 3, clon 2-España ${ }^{6 B}$ (patrón D). Carril 13, INS Spn E-226 serotipo 6B (patrón D). Carriles 6 a 9, 1727, 1995, 1822, 1232 con patrón D. Carriles 4 y 11, 1096 y 875 con patrón D1. Carriles 10 y 12, 1176 y 1178 con patrón D3. Carriles 5 y 14, 1675 patrón D2 y 787 patrón D4. B: dendrograma de aislamientos de los S. pneumoniae serotipo 6B. 


\section{Determinación de los perfiles de PspA}

Por medio de la amplificación por PCR del gen pspA, 33 (41,3\%) aislamientos presentaron productos de amplificación correspondientes con los de la familia 1, $43(53,7 \%)$ amplificaron con los iniciadores para la familia 2, y $4(4,9 \%)$ amplificaron para los genes de ambas familias. Por la técnica de dot-blot para la proteína PspA, 32 aislamientos se agruparon en la familia 1 $(39,5 \%), 45(55,6 \%)$ en la familia 2 , y 3 aislamientos reaccionaron con ambos anticuerpos. Al comparar las dos técnicas se obtuvo un porcentaje de concordancia de $92,5 \%$.

\section{Distribución de los clones según procedencia y diagnóstico}

Los datos indicaron que los clones internacionales 1, 2 y 3 circulaban en Antioquia y Bogotá. En Antioquia se recuperaron aislamientos genéticamente relacionados con los 4 clones, mientras que en Bogotá, el clon prevalente fue el $3(57 \%)$ con aislamientos del subtipo B3 y no se recuperaron aislamientos relacionados con el clon $25-C o l o m b i a^{23 F}$. A pesar del reducido número de aislamientos recuperados en los otros tres departamentos, se pudo establecer la circulación de, al menos, un clon internacional en ellos. Al relacionar los clones con el diagnóstico, los datos señalaron que $27 \%$ de los casos de meningitis fueron causados por aislamientos relacionados con el clon 3, $19 \%$ por el clon 25 y $15 \%$ por el clon 2. El $40 \%$ de las neumonías fueron ocasionadas por aislamientos relacionados con el clon 3, $13 \%$ por el clon 25 y $11 \%$ por el clon 2 .

\section{Discusión}

Los estudios de tipificación molecular han permitido identificar clones de $S$. pneumoniae resistentes a penicilina, algunos de los cuales presentan una dispersión global. En 1990, Muñoz y col. (30) demostraron la dispersión del clon 1España $^{23 F}$ a los Estados Unidos, el cual fue posteriormente identificado en diferentes regiones del mundo $(4,16,21,31)$. Varios estudios en Suramérica han establecido una baja frecuencia en la recuperación de aislamientos de $S$. pneumoniae relacionados con el clon 1 en población infantil (32-34), los cuales contrastan con los obtenidos en México, donde los aislamientos relacionados con este clon tienen una prevalencia de $66 \%$ (9), similar a la obtenida en los países asiáticos donde la resistencia a la penicilina asociada con el clon 1 supera $50 \%$ (4).

En Colombia, Castañeda y col. estudiaron por PFGE un grupo de 59 aislamientos invasores de S. pneumoniae y encontraron que $13,6 \%$ presentaban características electroforéticas comunes a las del clon 1 (20); un estudio posterior demostró que $8,9 \%$ de los aislamientos con SDP recuperados en niños menores de 5 años están relacionados con este clon (21). En nuestros resultados, sólo $2,5 \%$ de los aislamientos estaban relacionados con el clon 1-España ${ }^{23 F}$, tenían en común el perfil PBP I, las características fenotípicas de multirresistencia idénticas a las del clon y expresaban la proteína PspA de la familia 2. La clonalidad de los genes pbp en aislamientos $23 \mathrm{~F}$ con un mismo genotipo, pero de distinta procedencia, fue demostrada por Muñoz y col. (35) y la presencia del gen pspA familia 2 en el clon 1 por Beall y col. (17). Lo anterior aporta evidencia de la circulación del clon 1 en nuestro medio, tanto en la población infantil como en la adulta.

Desde los primeros estudios de caracterización molecular de aislamientos colombianos de $S$. pneumoniae se estableció la circulación del clon 25 -Colombia ${ }^{23 F}$ en la población infantil $(20,21)$. Nuestros resultados aportan evidencia de la presencia de este grupo clonal en la población adulta, en la cual representan $13,8 \%$ de los aislamientos estudiados que comparten un mismo patrón PFGE, perfil PBP II (82\%) y familia PspA 1 (91\%). Todos estos aislamientos presentaron resistencia intermedia a la penicilina y patrones únicos de restricción de los genes $p b p 2 x, 2 b$ y $1 a$, lo que sugiere una evolución independiente de estas formas de baja afinidad de las PBP, posiblemente debido a la presión selectiva por el antibiótico o a eventos de recombinación genética de los genes $p b p$, los cuales, una vez formados, se han establecido a través del tiempo (36).

Por medio de PFGE, Moissenett y col. observaron que algunos aislamientos serotipo 14 estaban estrechamente relacionados con los patrones de restricción del clon serotipo $9 \mathrm{~V}$ español y 
demostraron que aislamientos con diferente tipo capsular pueden estar relacionados genéticamente (37). A este clon se le denominó clon 3-España9v, con el serotipo 14 como su principal variante $(13,37)$. El clon 3-España ${ }^{9 v}$ es altamente prevalente en los aislamientos con SDP recuperados de niños menores de 5 años en países suramericanos $(21,32-34)$ y se recupera con menor frecuencia en México y Estados Unidos $(9,38)$. Esto ha permitido establecer una distribución regional de los clones 1 y 3 ; el clon 1-España ${ }^{23 F}$ es más prevalente en la zona norte de América, mientras que el clon 3- España ${ }^{9 v}$ se recupera con mayor frecuencia en la parte sur del continente (38).Según los patrones electroforéticos obtenidos en nuestro estudio, 83,3\% de los aislamientos serotipo 14 y $71,5 \%$ del $9 \mathrm{~V}$ están relacionados con el clon 3. El $40 \%$ de estos aislamientos expresaron el perfil PBP I encontrado en el clon 1, un ejemplo de la dispersión horizontal de determinantes de resistencia en $S$. pneumoniae como resultado de la transformación natural $(36,39)$. El perfil PBP III se encontró en $50 \%$ de los aislamientos, principalmente en los del subtipo $\mathrm{B} 3$, y tenían un patrón de restricción del gen pbp $2 \mathrm{~b}$ diferente al observado en el perfil I, resultado de eventos de recombinación genética que alteran el sitio de reconocimiento de la enzima pero no afectan el fenotipo de resistencia (15). Además, $93 \%$ de estos aislamientos expresaron la proteína de superficie A de la familia 2; esta similitud los relaciona con el clon 3 -España ${ }^{9 v}$ y sugiere un ancestro evolutivo común $(17,35)$.

El fenotipo del clon 2-España ${ }^{6 B}$ fue identificado en España y a finales de los años 80 llegó a Islandia donde se estableció como el clon dominante (40). En América se han informado aislamientos relacionados con el clon 2 en Colombia, Chile, Estados Unidos y Brasil $(21,33,38,41)$. Todos los aislamientos que expresaron el tipo capsular 6B, se encontraron relacionados por PFGE, patrones de resistencia a antimicrobianos y expresión de PspA con el clon 2-España ${ }^{6 B}$. Los perfiles de PBP fueron muy heterogéneos en este grupo clonal, similar a lo observado en los aislamientos 6B recuperados de niños (21). No obstante, se pudieron establecer ciertas subpoblaciones clonales; un primer grupo tenía el perfil PBP VI y un segundo grupo, el perfil PBP VII.
Los aislamientos con otros tipos capsulares no se relacionaron electroforéticamente con clones internacionales, presentaron un alto polimorfismo de los genes pbp y la distribución de la proteína PspA fue equivalente para ambas familias. Lo anterior indica que la variabilidad observada por tipificación capsular se relaciona con la heterogeneidad genética de los aislamientos (40). En estos aislamientos se observó la transferencia horizontal de genes $p b p$ de $S$. pneumoniae a aislamientos con diferente tipo capsular; el perfil PBP II característico del clon 25-Colombia ${ }^{23 F}$ se encontró en dos aislamientos serotipo 15B y 19B no relacionados electroforéticamente con el clon y el perfil XVI en 4 aislamientos con diferente serotipo y patrón PFGE. Se puede asumir que han adquirido resistencia a la penicilina por medio de eventos genéticos independientes a través de transformación genética o mutaciones en los genes pbp (35). Además, estos aislamientos pueden representar la emergencia y dispersión de nuevos grupos clonales que enfatizan la importancia de los programas de vigilancia epidemiológica.

Los resultados de tipificación molecular determinaron la presencia y circulación de los clones internacionales 1, 2, 3 y 25 como agentes causantes de meningitis y neumonía en la población adulta, especialmente del clon 3España ${ }^{9 v}$, el cual se aisló de $40 \%$ de los pacientes con diagnóstico de neumonía. Estos clones internacionales representan $52,5 \%$ de los aislamientos resistentes a la penicilina y se recuperaron de pacientes en diferentes lugares geográficos de nuestro país, principalmente de Bogotá (48\%) y el departamento de Antioquia (33\%), posiblemente debido a que son las zonas con mayor población y económicamente más activas, donde la industria y el turismo estimulan constantemente la presencia de viajeros locales e internacionales, de forma tal que la densidad poblacional favorece la prevalencia del clon, los procesos migratorios, su diseminación y la eficiencia del sistema de salud, la recuperación del aislamiento.

Las altas tasas de intercambio genético por recombinación entre estas bacterias naturalmente transformables, la dispersión de clones resistentes 
y la introducción de una nueva vacuna en el mercado, que pueden afectar la prevalencia de los clones existentes y seleccionar nuevos grupos clonales con ventajas adaptativas, hacen necesario mantener el sistema de vigilancia epidemiológica tanto del fenotipo como del genotipo de S. pneumoniae en nuestra población. Además, el conocimiento de la prevalencia y dispersión de los grupos clonales involucrados más frecuentemente con el desarrollo de infecciones y resistencia a antimicrobianos permite concentrar esfuerzos tendientes a diseñar nuevas estrategias preventivas para el control de la enfermedad neumocócica y disminuir así tanto su morbilidad como la mortalidad.

\section{Agradecimientos}

Al personal del Grupo de Microbiología del Instituto Nacional de Salud por su colaboración; a María Claudia Vela por su aporte en el desarrollo de las técnicas moleculares; a Antonio Mejía, gerente de AM Ltda. y representante de Bio-Rad Life Science, por permitirnos usar el programa Diversity y a Colciencias por la cofinanciación de este proyecto a través del programa de jóvenes investigadores (convenio 144/2001).

\section{Referencias}

1. Boulnois GJ. Pneumococcal proteins and pathogenesis of disease caused by Streptococcus pneumoniae. J Gen Bacteriol 1992;138:249-59.

2. Fedson DS, Antthony J, Scott JA. The burden of pneumococcal disease among adults in developed and developing countries: what is and is not known. Vaccine 1999;17(Suppl.1):S11-S18.

3. Austrian R. The pneumococcus at the millennium: not down, not out. J Infect Dis 1999;179 (Suppl 2):S338-41.

4. Song J-H, Lee NY, Ichiyama S, Yoshida R, Hirakata Y, Fu, W, et al. Spread of drug-resistant Streptococcus pneumoniae in Asian countries: Asian network for surveillance of resistant pathogens (ANSORP) study. Clin Infect Dis 1999;28:1206-11.

5. Baquero F, García-Rodríguez JA, García de Lomas J, Aguilar L, and the Spanish Surveillance Group for Respiratory Pathogens. Antimicrobial resistance of 1,113 Streptococcus pneumoniae isolates from patients with respiratory tract infections in Spain: results of a 1-year (1996-1997) multicenter surveillance study. Antimicrob Agents Chemother 1999;43:357-9.

6. Doern Gary V. Antimicrobial use and the emergence of antimicrobial resistance with Streptococcus pneumoniae in the United States. Clin Infect Dis 2001;33(Suppl.3):S18792.

7. Greenberg D, Speert DP, Mahenthiralingam E, Henry DA, Campbell NE, Scheifele DW and the CPS/LCDC IMPACT Monitoring Network. Emergence of penicillinnonsusceptible Streptococcus pneumoniae invasive clones in Canada. J Clin Microb 2002;40:68-74.

8. De Cunto MC, Di Fabio JL, Dias VS, Cobo R, Casagrandre ST, Pignatari AC, et al. Geographic distribution of penicillin resistance of Streptococcus pneumoniae in Brazil: genetic relatedness. Microb Drug Resist 1998;4:209-17.

9. Echániz-Aviles G, Velázquez ME, Carnalla MN, Soto A, Di Fabio JL, Solorzano F, et al. Predominance of multiresistant 23F international clonal of Streptococcus pneumoniae among isolates from Mexico. Microb Drug Resist 1998;4:241-6.

10. Agudelo $\mathrm{Cl}$, Sanabria O, Ovalle MV, Castañeda E y el Grupo Colombiano de trabajo en Streptococcus pneumoniae. Vigilancia por el laboratorio de Streptococcus pneumoniae aislado de procesos invasores en niños menores de 5 años: actualización de los datos 1994-2000. Inf Quinc Epidem Nac 2001;6:99-104.

11- Dessen A, Mouz N, Gordon E, Hopkins J, Didebergb O. Crystal structure of PBP $2 x$ from highly penicillinresistant Streptococcus pneumoniae clinical isolates. J Biol Chem 2001;276:106-12.

12. Hakenbeck R, Kaminski K, König A, Linden MVD, Paik J, Reichmann P, et al. Penicillin-binding proteins in b-lactam-resistan Streptococcus pneumoniae. Microb Drug Resist 1999;5:91-9.

13. Madein MCJ. Horizontal genetic exchange, evolution, and spread of antibiotic resistance in bacteria. Clin Infect Dis 1998;27:S12-20.

14. McGee L, McDougal L, Zhou J, Spratt G, Tenover FC, George R, et al. Nomenclature of major antimicrobial-resistant clones of Streptococcus pnemunoniae defined by the pneumococcal molecular epidemiology network. J Clin Microb 2001;39:2565-71.

15. Lefevre JC, Faucon G, Sicard AM, Gasc AM. DNA fingerprinting of Streptococcus pneumoniae strains by pulsed-field gel electrophoresis. J Clin Microb 1993;31: 2724-8.

16. Hall LMC. Application of molecular typing to the epidemiology of Streptococcus pneumoniae. J Clin Pathol 1998; 51:270-4.

17. Beall B, Gherardi G, Facklman RR, Hollingshead SK. Pneumococcal pspA sequence types of prevelent multirresistant pneumococcal strains in the United States and of internationally disseminated clones. J Clin Microbiol 2000;38:3663-9.

18. Crain MJ, Hollingshead SK, MacDaniel LS. Pneumococcal diversity: considerations for new vaccine strategies with emphasis on pneumococcal surface protein $A$ (PspA). Clin Microb Rev 1998;11:645-57. 
19. Hollingshead SK, Becker R, Briles DE. Diversity of PspA: mosaics genes and evidence for past recombination in Streptococcus pneumoniae. Infect Immun 2000; 68:5889-900.

20. Castañeda E, Peñuela I, Vela C, Tomasz A. Penicillinresistant Streptococcus pneumoniae in Colombia: presence of international epidemic clones. Microb Drug Resist 1998;4:233-6.

21. Vela MC, Fonseca N, Di Fabio JL, Castañeda E. Presence of international multiresistant clones of Streptococcus pneumoniae in Colombia. Microb Drug Resist 2001;7:153-64.

22. Vela MC, Fonseca N, Castañeda E, Di Fabio JL, Hollingshead F, Briles DE, et al. Pneumococcal surface protein A of invasive Streptococcus pneumoniae isolates from Colombian children. Emerg Infect Dis 2001;7:832-6.

23. Agudelo CI, Díaz PL, Sanabria OM, Ovalle MV, Castañeda E y el Grupo colombiano de trabajo en Streptococcus pneumoniae. Vigilancia por el laboratorio de Streptococcus pneumoniae aislado de procesos invasores en población mayor de 5 años: actualización de los datos 1998-2001. Inf Quinc Epidem Nac 2002;7:177-83.

24. National Committe for Clinical Laboratory Standards (NCCLS). Performance standards for antimicrobial susceptibility testing: ninth information supplement. NCCLS document. Vol 18, no.1. Wayne, PA: National Committe for Clinical Laboratory Standards; 1999.

25. Soares S, Kristionsson KG, Musser JM, Tomasz A. Evidence for introduction of a multiresistant clone of serotype 6B Streptococcus pneumoniae from Spain to Iceland in late 1980s. J Infect Dis 1993;168:158-63.

26. Tenover FC, Arbeit RD, Gaering RV, Mickelsen PA, Murray BE, Persing DH, et al. Interpreting chromosomal DNA restriction patterns produced by pulsed-field gel electrophoresis: criteria for bacterial isolate typing. J Clin Microb 1995;33:2233-9.

27. Pitcher DG, Saunders NA, Owen RJ. Rapid extraction of bacterial genomic DNA with guanidium thyocyanate. Lett Appl Microbiol 1989;4:225-31.

28. Briles DE, Creech E, Swiartlo JP, Dillard P, Smith KA, Benton B, et al. Pneumococcal diversity: considerations for new vaccine strategies with emphasis on pneumococal surface protein A (PspA). Clin Microb Rev 1998;11:645-57.

29. Smith PK, Krohn RI, Hermanson GT, Mallia AK, Gartner FH, Provenzano MD, et al. Measurement of protein using bicinchonic acid. Anal Biochem 1985;150: 76-85.

30. Muñoz R, Coffey TJ, Daniels M, Dowson CG, Laible G, Casal J, et al. Intercontinental spread of a multiresistant clone of serotype 23F Streptococcus pneumoniae. $\mathrm{J}$ Infect Dis 1991;164:302-6.
31. Hermans PW, Sluijter M, Dejsirilert S, Lemmens N, Elezenaar K, Veen AV et al. Molecular epidemiology of drug-resistant pneumococci: towards an international approach. Microb Drug Resist 1997;3:243-51.

32. Camou T, Hortal M, Tomasz A. The apparent importation of penicillin-resistant capsular type 14 Spanish/French clone of Streptococcus penumoniae into Uruguay in early 1900s. Microb Drug Resist 1998;4:219-24.

33. Gherardi G, Inostrozo JS, O'Ryan M, Prado V, Prieto $\mathrm{S}$, Arellano C, et al. Genotypic survey of recent blactam-resistant pneumococcal nasopharyngeal isolates from asymptomatic children in Chile. J Clin Microb 1999; 37:3725-30

34. Rossi A, Corso A, Pace J, Regueira M, Tomasz A. Penicillin-resistant Streptococcus pneumoniae in Argentina: frequent occurrence of an internationally spread serotype 14 clone. Microb Drug Resist 1998;4: 225-31.

35. Muñoz R, Musser JM, Crain M, Briles DE, Marton A, Parkinson AJ, et al. Geographic distribution of penicillin-resistant clones of Streptococcus pneumoniae: characterization by penicillin-binding protein profile, surface protein A typing, and multilocus enzyme analysis. Clin Infec Dis 1992;15:112-28.

36. Coffey TJ, Dowson C, Daniels M, Zhou J, Martin C, Spratt G, et al. Horizontal transfer of multiple penicillinbinding protein genes, and capsular biosynthetic genes, in natural populations of Streptococcus pneumoniae. Mol Microb 1991;5:2255-60.

37. Moissenet D, Valcin M, Marchand V, Garadéian E, Gesli P, Garbarg-Chenon A, et al. Molecular epidemiology of Streptococcus pneumoniae to penicillin in a Paris children's hospital. J Clin Microb 1997;35:298-301.

38. Ritcher SS, Heilmann KP, Coffman SL, Huynh HK, Brueggemann AB, Pfaller MA, et al. The molecular epidemiology of penicillin-resistant Streptococcus pneumoniae in the United States, 1994-2000. Clin Infect Dis 2002;34:330-9.

39. Doit C, Denamur E, Picard B, Gelsin P, Elion J, Bingen E. Mechanisms of the spread of penicillin resistance in Streptococcus pneumoniae strains causing meningitis in children in France. J Infect Dis 1996;174:520-8.

40. Vilhelmsson S E, Tomasz A, Kristinsson KG. Molecular evolution in a multidrug-resistant lineage of Streptococcus pneumoniae: emergence of strains belonging to the serotype $6 \mathrm{~B}$ Icelandic clone that lost antibiotic resistance traits. J Clin Microbiol 2000; 38:1375-81.

41. Wolf B, Rey LC, Brisse S, Moreira LB, Milatovic D, Fleer A, et al. Molecular epidemiology of penicillinresistant Streptococcus pneumoniae colonizing children with community-acquirired pneumonia and children attending day-care centres in Fortaleza, Brazil. J Antimicrob Chemother 2000;46:757-65. 